\title{
Ultra-wideband Propagation Loss Around a Human Body in Various Surrounding Environments
}

\author{
H. Yamamoto and T. Kobayashi
}

\begin{abstract}
Ultra-wideband (UWB) technologies have been anticipated for use in wireless body area networks (WBAN) because of their low power consumption and anti-multipath capabilities. This chapter presents the UWB $(3.1-10.6 \mathrm{GHz})$ propagation loss in WBAN scenarios between on-body antennas in three different surrounding environments. The measurements were performed in a 3-m radio anechoic chamber, a classroom, and a small room. The propagation paths were roughly divided into line-of-sight (LOS) and non-LOS (NLOS) ones. Small rooms, particularly NLOS, yielded higher reception power than larger rooms. This was attributed to the ample multipath from the nearby floor, walls, and ceiling. The UWB maximum propagation losses in three surrounding environments were smaller than ones of $\mathrm{CW}(6.85 \mathrm{GHz})$. This is because nulls caused by interference were cancelled out by the ultra-wide bandwidth. The propagation losses of low-band $(3.4-4.8 \mathrm{GHz})$ and high-band (7.25-10.25 GHz) UWB were also evaluated. In WBAN scenarios, the low-band yielded lower propagation loss than the high-band and approximately the same loss as the full-band UWB $(3.1-10.6 \mathrm{GHz})$.
\end{abstract}

Keywords Ultra-wideband (UWB) · Wireless body area networks · Radio propagation $\cdot$ Propagation loss $\cdot$ Multipath propagation

\section{Introduction}

Wireless body area networks (WBAN) have been discussed for medical and non-medical applications [1]. For medical applications, wireless electroencephalography (EEG), electrocardiography (ECG), electromyography (EMG), and other health-care monitoring are proposed applications. Ultra-wideband (UWB) technologies were anticipated for use in WBAN because of their low power consumption and anti-multipath capabilities. In the last few years, researchers investigated UWB indoor and outdoor radio propagation modeling and characterization [2, 3]. A number of measurements were also carried out to characterize on-body UWB propagation in the WBAN scenarios [4]. However, previous WBAN studies treated mainly the cases when propagation was measured in either a radio anechoic chamber or a particular room [5-7]. It is necessary to evaluate the variation of propagation losses in various surrounding environments from the viewpoint of WBAN device design.

H. Yamamoto $(\bowtie)$

Tokyo Denki University, Tokyo, Japan

e-mail: h.yamamoto@grace.c.dendai.ac.jp 
This chapter presents the UWB (3.1-10.6 GHz) propagation loss in WBAN scenarios between onbody antennas in three surrounding environments: a 3-m radio anechoic chamber, a classroom, and a small room. The propagation losses of continuous wave $(\mathrm{CW})$, low-band UWB (3.4-4.8 GHz), and high-band UWB (7.25-10.25 GHz) were evaluated in comparison with that of full-band UWB $(3.1-10.6 \mathrm{GHz})$.

\section{Experimental Setup}

The measurements were carried out in the following rooms:

\begin{tabular}{lllll}
\hline & Width $(\mathrm{m})$ & Depth $(\mathrm{m})$ & Height $(\mathrm{m})$ & Volume $\left(\mathrm{m}^{3}\right)$ \\
\hline Anechoic chamber & 5.5 & 7.0 & 5.0 & 193 \\
Classroom & 6.6 & 4.3 & 2.5 & 71 \\
Small room & 2.6 & 2.7 & 2.3 & 16 \\
\hline
\end{tabular}

The 3-m radio anechoic chamber can be considered as a room extending with an infinite volume in terms of radio propagation. The classroom and the small room were made of reinforced concrete, and their floors, walls, and ceilings were covered with linoleum, wall paper, and plaster board, respectively. A volunteer (an adult male, $1.77 \mathrm{~m}$ tall and $57 \mathrm{~kg}$ ) stood upright with legs close together in either a quiet zone of the radio anechoic chamber or the center of the rooms. The UWB

Fig. 1 Placement of the transmitting and receiving antennas on the body.

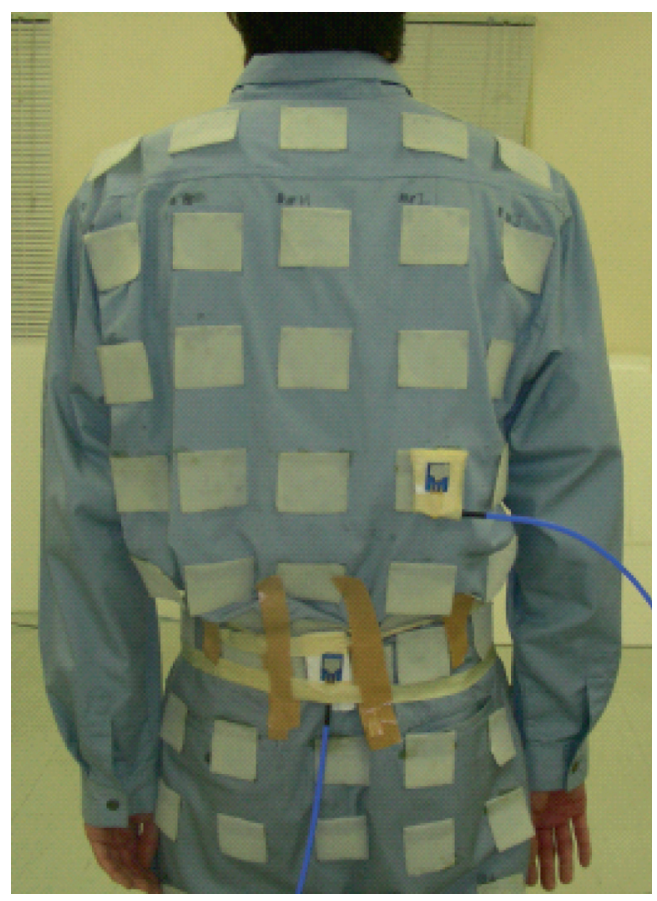


(from 3.1 to $10.6 \mathrm{GHz}$ ) frequency- and time-domain propagations losses were measured with a vector network analyzer. Commercially available meanderline antennas [8] were used for transmission and reception. The voltage standing wave ratio of the meanderline antenna was less than 2.5 from 3.1 to $10.6 \mathrm{GHz}$ and the omnidirectionality in the horizontal plane was within $3 \mathrm{~dB}$ in a free space. The transmitting antenna was fixed on the center back waist of the volunteer placed at a height of $1 \mathrm{~m}$ from the floor, as shown in Fig. 1 (also indicated by * in Fig. 2). The receiving antenna was placed at 100-mm intervals on the body. Both antennas were vertically polarized and separated $10 \mathrm{~mm}$ apart from the body surface. When the receiving antenna was placed on the back of the body, the path was roughly line-of-sight (LOS); when in the front, it was non-LOS (NLOS). In total 153 receiving points around the human body were employed. The transmitting and receiving antennas were fed by 2 and 3-m long coaxial cables and these cables were perpendicular in configuration without crossing to reduce unwanted cable coupling [9].

\section{Measurement Results}

The UWB propagation losses were calculated by integrating the power of the losses between the feeding points of the antennas over the occupied bandwidth. The calibration was conducted between the feeding points with a coaxial through adaptor. These propagation losses embraced the antenna

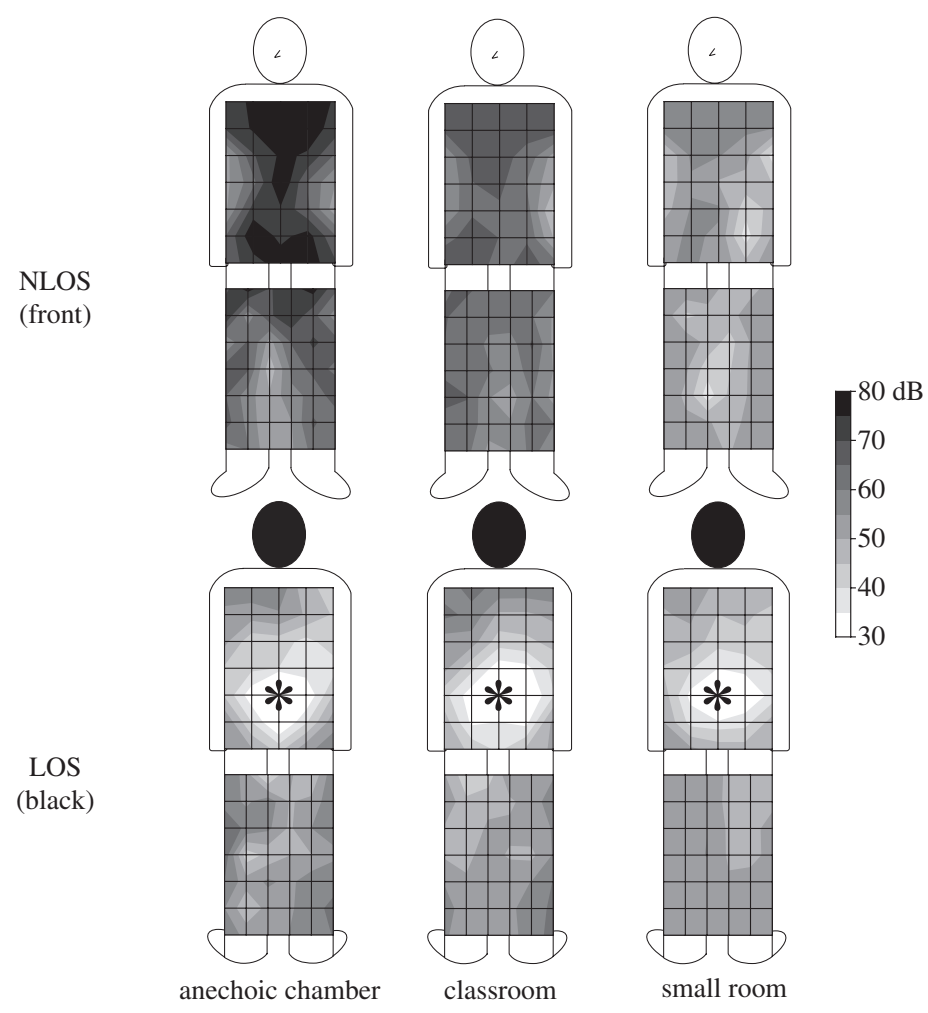

Fig. 2 The distributions of UWB propagation losses in three environments (the transmitting antenna was placed at a point denoted by ${ }^{*}$ ) 

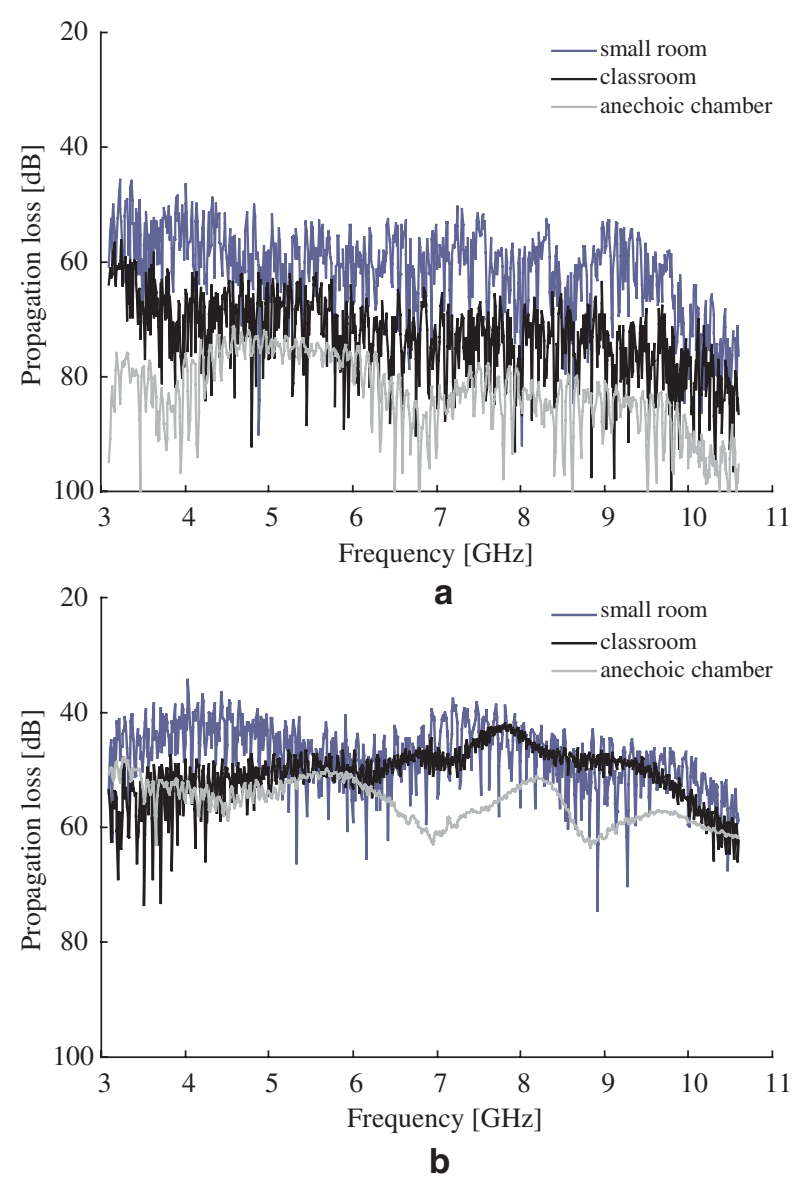

Fig. 3 Frequency-domain propagation losses at (a) the center of the chest (NLOS) and (b) the center of the back (LOS)

gains (typically -10 to $3 \mathrm{dBi}$ ), which were affected by the human body and depended on the radiation direction and the frequency [10]. The distributions of UWB propagation losses are shown in Fig. 2. The small room yielded higher reception power than larger rooms, particularly for NLOS. Examples of the frequency- and time-domain propagation losses on the front side of the center chest and the back side of one (receiving antenna was placed at a height of $1300 \mathrm{~mm}$ from the floor) are shown in Figs. 3 and 4. The propagation losses in the radio anechoic chamber and the small room were the highest and the lowest, respectively, whereas the classroom was in between, as shown in Fig. 3. This was attributed to the ample multipath from the nearby floor, walls, and ceiling in the classroom and small room, as shown in Fig. 4. The propagation path of the radio anechoic chamber was either direct or diffract wave, and thus the reception power is low. Multipath in the small room resulted in the lowest path loss because the propagation lengths were shorter than in the classroom, and therefore the total received power was the highest.

The propagation losses of the CW $(6.85 \mathrm{GHz})$, low-band UWB, and high-band UWB were evaluated in comparison with full-band UWB. The maximums, minimums, and medians of UWB, CW, low-band UWB, and high-band UWB propagation losses measured in the three environments were derived, as shown in Fig. 5. The variation ranges (between the minimums and the maximums) in 

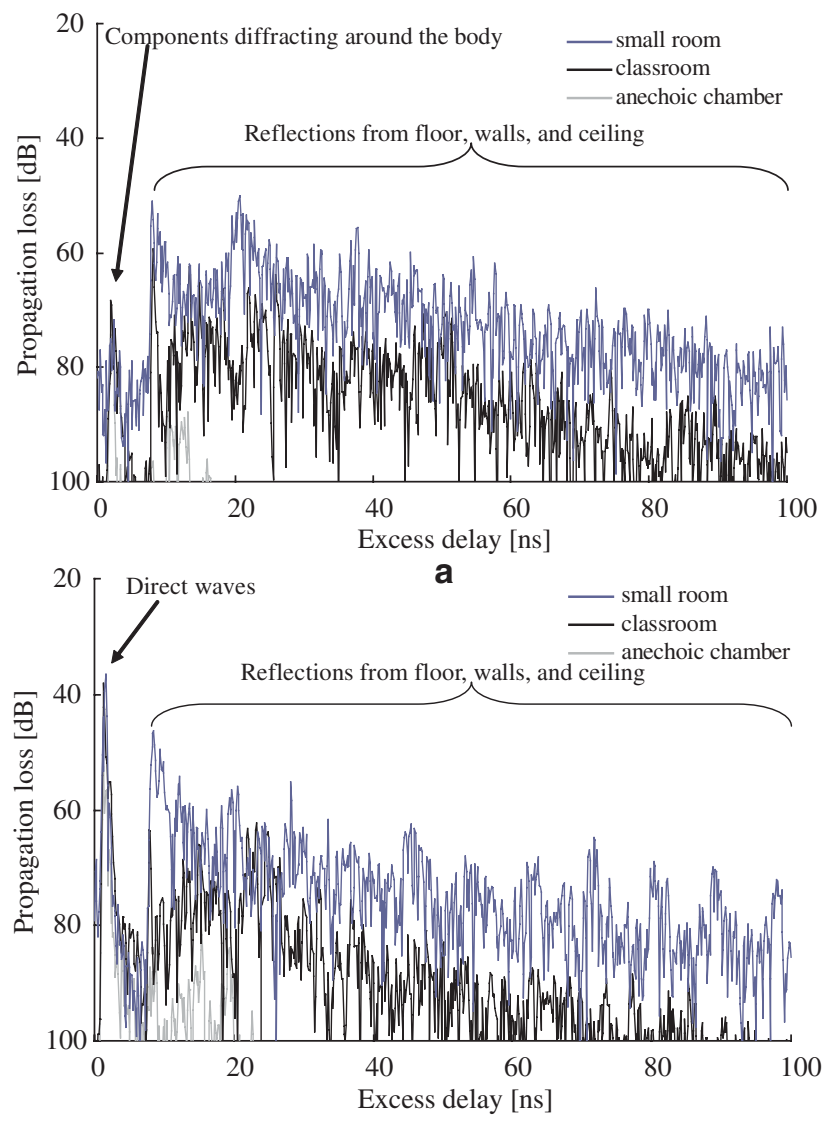

b

Fig. 4 Time-domain propagation losses at (a) the center of the chest (NLOS) and (b) the center of the back (LOS)

UWB were smaller than ones in CW (in particular, more than $10 \mathrm{~dB}$ smaller in NLOS). This is because nulls caused by interference were cancelled out by the ultra-wide bandwidth. The results indicate that UWB technologies are more advantageous than narrowband ones from the viewpoint of reducing fading margins, although frequency-selective fading inevitably takes place. The variation ranges in full- and low-band UWB were similar for both NLOS and LOS. The maximum propagation losses of the high-band UWB, in particular radio anechoic chamber, yield $17 \mathrm{~dB}$ (at LOS) and $11 \mathrm{~dB}$ (at NLOS) higher than that of full-band UWB. The low-band UWB is hence more advantageous than high-band UWB in terms of receiving high reception power, if the full-band UWB is not legalized (e. g., in Japan and Europe at present).

\section{Conclusions}

The UWB propagation losses were measured between on-body antennas in three different surrounding environments envisioned for WBAN scenarios. Small rooms yielded higher reception power than larger rooms. This was attributed to the ample multipath from the nearby floor, walls, and 

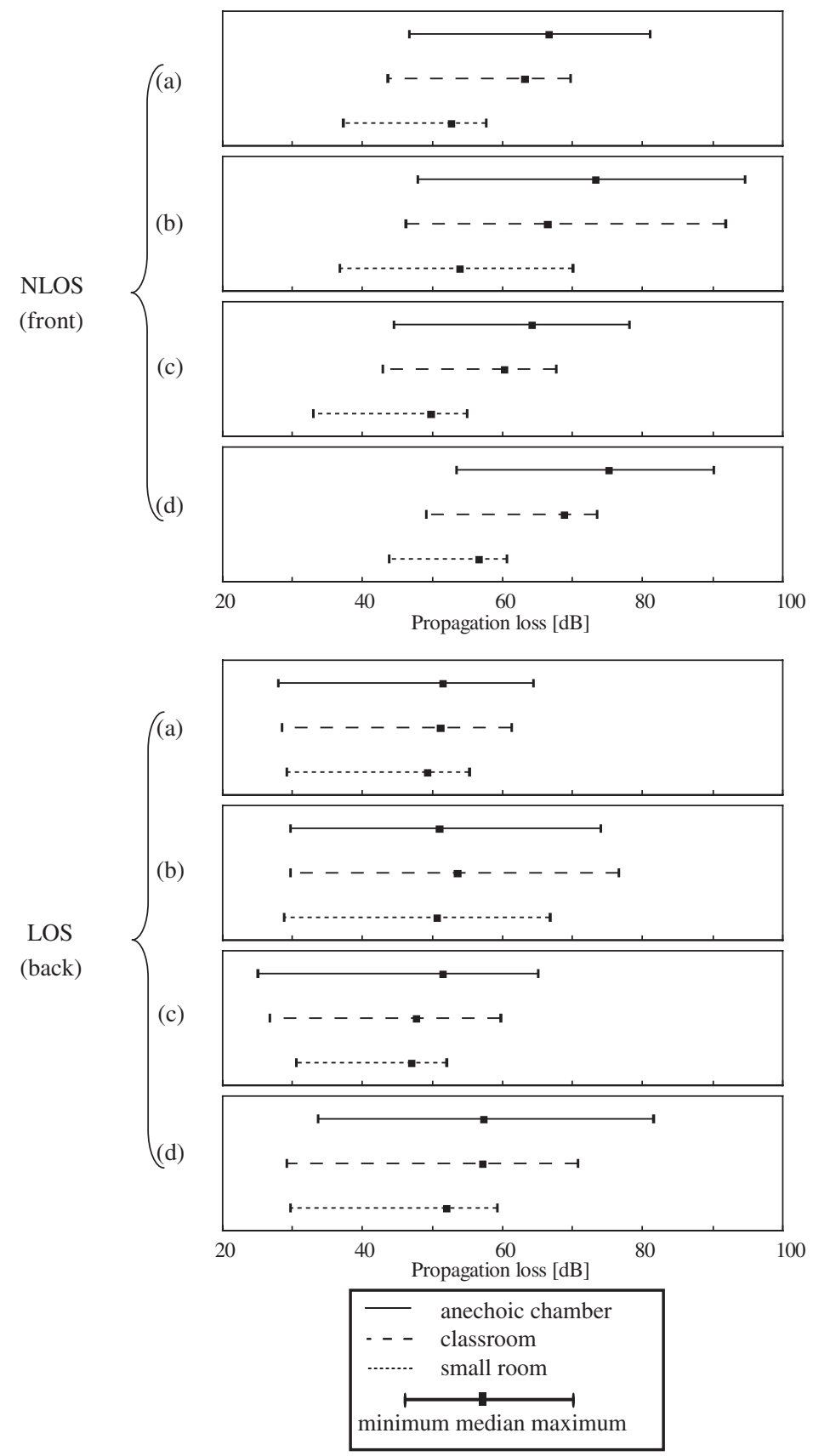

Fig. 5 The maximums, minimums, and medians of propagation losses in three environments for (a) UWB (3.1$10.6 \mathrm{GHz}),(\mathbf{b}) \mathrm{CW}(6.85 \mathrm{GHz})$, (c) low-band UWB (3.4-4.8 GHz), and (d) high-band UWB (7.25-10.25 GHz) 
ceiling. The UWB maximum propagation losses in three environments were smaller than that of $\mathrm{CW}$. This is because nulls caused by interference were cancelled out by the ultra-wide bandwidth. The propagation losses of low-band UWB and high-band UWB were also evaluated. In WBAN scenarios, low-band UWB is more advantageous than high-band UWB from the viewpoint of reducing propagation losses, if the usage of the full-band UWB is legally banned.

Acknowledgments The authors would like to thank the members of the Medical-ICT Group, National Institute of Information and Communications Technology, Japan, for valuable discussion, and in particular Shunsuke Sato (now with Sanyo Electric) for valuable help in the experiments and discussions.

\section{References}

1. J. Ryckaert, B. V. Poucke, B. Gyselinckx, and S. Donnay, Wireless body area networks, ftp://ieee:wireless@ftp.802wirelessworld.com/15/03/15-03-0484-00-004a-sg4a-cfa-response-wireless-bodyarea-networks.ppt, 7 November, 2003.

2. C. Chang, Y. Kim, and S. Lee, UWB indoor propagation channel measurements and data analysis in various types of high-rise apartments, in Proceedings 2004 IEEE 60th Vehicular Technology Conference (VTC2004-Fall), Los Angeles, California, USA, vol. 1, pp. 150-4, 26-29 September 2004.

3. S. S. Ghassemzadeh, R. Jana, C. W. Rice, W. Turin, and V. Tarokh, A statistical path loss model for in-home UWB channels, in Proceedings 2002 IEEE Conference on Ultra Wideband Systems and Technologies (UWBST 2002), Baltimore, USA, pp. 59-64, 21-23 May 2002.

4. P. S. Hall and Y. Hao (eds.), Antennas and Propagation for Body-Centric Wireless Communications, Artech House, Boston, MA, 2006.

5. A. Alomainy, Y. Hao, X. Hu, C. G. Parini, and P. S. Hall, UWB on-body radio propagation and system modeling for wireless body-centric networks, IEE Proceedings Communications, vol. 153, no. 1, pp. 107-14, February 2006.

6. T. Zasowski, F. Althaus, M. Stager, A. Wittneben, and G. Troster, UWB for noninvasive wireless body area networks: channel measurements and results, in Proceedings 2003 IEEE Conference on Ultra Wideband Systems and Technologies (UWBST 2003), Reston, Virginia, USA, pp. 285-9, 16-19 November 2003.

7. A. Fort, J. Ryckaert, C. Desset, P. D. Doncker, P. Wambacq, and L. V. Biesen, Ultra-wideband channel model for communication around the human body, IEEE Journal on Selected Areas in Communications, vol. 24, no. 4, pp. 927-933, April 2006.

8. Skycross, Inc., Antenna Products, 3.1-10 GHz ultra-wideband antenna, http://www.skycross.com/Products/ PDFs/SMT-3TO10M-A.pdf.

9. H. Yamamoto and T. Kobayashi, Effects of feeding cable configurations on propagation measurements between small ultra wideband antennas for WBAN applications, in International Workshop on Future Wellness and Medical ICT Systems (FEELIT2008), Lapland, Finland, 9 September 2008, to be published.

10. H. Yamamoto and T. Kobayashi, Measurements and characterization of ultra wideband propagation channels between a base station and on-body antennas, in Second International Symposium on Medical Information and Communication Technology (ISMICT2007), Oulu, Finland, 11-13 December 2007. 